\title{
Reliability and Validity of the Early Trauma Inventory Self Report-Short Form among Korean Adolescents
}

\author{
Subin Park \\ Department of Research Planning, Mental Health Research Institute, National Center for Mental Health, Seoul, Korea
}

Objectives: Experiencing early childhood trauma is related to multiple psychiatric problems in adolescents and adulthood. This study aimed to examine the reliability and validity of the Korean version of the Early Trauma Inventory Self Report-Short Form (ETISR-SF) among Korean adolescents.

Methods: A total of 86 adolescents aged 12-17 years (mean age 14.50 \pm 1.35 years, range 12-17) were assessed using the ETISR-SF. Other instruments, including the Children's Depression Inventory (CDI), the revised Children's Manifest Anxiety Scale (RCMAS), and the List of Threatening Experiences Questionnaire (LTE-Q), were used to assess clinical symptoms. After 2 months, 51 of the 86 participants were evaluated using the ETISR-SF to assess test-retest reliability.

Results: The Cronbach's coefficient alpha for the ETISR-SF was high (0.803). Adolescents with depressive disorder showed higher ETISR-SF scores compared to healthy controls. The ETISR-SF scores were correlated higher with the scores on the LTE-Q ( $r=0.485$ ) than with the scores on the CDI or RCMAS ( $r=0.165$ and 0.347 , respectively).

Conclusion: The ETISR-SF was temporally stable, showing acceptable reliability $(r=0.776)$. These findings suggest that the Korean version of the ETISR-SF appears to be a reliable and valid instrument for the measurement of reported childhood trauma.

Key Words: Early trauma; Trauma inventory; Adolescent; Korea.

Received: June 5, 2017 / Revision: June 23, 2017 / Accepted: June 30, 2017

Address for correspondence: Subin Park, Department of Research Planning, Mental Health Research Institute, National Center for Mental Health, 127 Yongmasan-ro, Gwangin-gu, Seoul 04933, Korea

Tel: +82-2-2204-0108, Fax: +82-2-2204-0280, E-mail: subin-21@hanmail.net

\section{INTRODUCTION}

Childhood trauma is defined as an adverse childhood experience, including physical, emotional, and sexual abuse and general traumatic experiences. ${ }^{1)}$ Significant associations between childhood trauma and impaired mental health in adolescence $^{2-7)}$ and adulthood ${ }^{8-14)}$ have been documented in numerous studies. Early life experiences of trauma may affect children's ability to modulate physiological arousal, and the subsequent loss of self-regulation is related to both emotional problems such as depression and anxiety and behavioral problems such as self-destructive behavior and substance abuse. ${ }^{15)}$ Post-traumatic stress disorder (PTSD), anxiety, depression, eating disorders, sleep disorders, suicide attempts, conduct disorder, substance abuse, and internet addiction are among the psychiatric problems found to be associated with childhood trauma in previous studies. ${ }^{2-7,16-19)}$ There has also been a growing literature indicating that poly-victimization (exposure to more than one type of trauma) increases the

This is an Open Access article distributed under the terms of the Creative Commons Attribution Non-Commercial License (http://creativecommons.org/licenses/by-nc/4.0) which permits unrestricted non-commercial use, distribution, and reproduction in any medium, provided the original work is properly cited. risk of several psychiatric disorders and symptoms, such as anxiety, depression, PTSD, and suicide attempts. ${ }^{12-14,16,20,21)}$

Despite the significance of trauma, recalling trauma without specific questionnaires could limits the range of traumatic events. Therefore, a structured and comprehensive instrument to measure traumatic events is needed in both the clinical and research settings. There are numbers of instruments that assess developmental trauma in children and adolescents, and each of these instruments measure somewhat different dimensions of the childhood trauma. ${ }^{22)}$ However, there have been no studies validating these instruments with the Korean adolescent samples.

The Early Trauma Inventory Self Report-Short Form (ETISR-SF), one of instruments that measure the childhood trauma, is a 27-item self-report questionnaire, which assesses the four domains of physical, emotional, sexual abuse, and general trauma. ${ }^{23)}$ It was validated among the Korean adult samples, ${ }^{24)}$ but not among the Korean adolescent samples. Therefore, this study examined the reliability and validity of the Korean version of the ETISR-SF among Korean adolescent samples. 


\section{METHODS}

\section{Participants and procedure}

Forty adolescents aged $12-17$ years with a primary diagnosis of depressive disorder based on the Diagnostic and Statistical Manual of Mental Disorders, 4th edition, text revision were enrolled in this study. These psychiatric diagnoses were confirmed by the Korean Kiddie Schedule for Affective Disorders and Schizophrenia-Present and Lifetime version (K-SADS-PL). ${ }^{25)}$ Forty-six healthy adolescents aged $12-17$ years were also recruited from a middle school and a high school near our research center. All of them were screened for depression and other psychiatric disorders using the Korean version of the K-SADS-PL. To assess the test-retest reliability, the ETIR-SF was administered to participants two times (i.e., baseline and 2 months after baseline). This study was approved by the Institutional Review Board for human subjects (No. 2014-34). Informed consent was obtained from all participants.

\section{Measures}

\section{The Early Trauma Inventory Self-Report-Short Form}

The ETISR-SF is a 27-item questionnaire used for the assessment of the four domains of physical (5 items), emotional (5 items), and sexual (6 items) abuse, as well as general traumatic experiences (11 items). It categorically assesses the existence of traumatic events that occurred before the age of $18 .^{24)}$ Each traumatic experience was scored dichotomously (yes $=1 / \mathrm{no}=0$ ). The sum of scores of each domain and the total scores were calculated. The measure has been shown to have excellent validity and internal consistency among Korean adult population (Cronbach's $\alpha=0.869$, testretest reliability $=0.844) .{ }^{24)}$

\section{Exposure to life-threatening experiences}

A modified version of the List of Threatening Experiences questionnaire (LTE-Q) ${ }^{26)}$ was used to assess exposure to life-threatening experiences. The LTE-Q is a 12 -item instrument to measure common life events that tend to be threatening. The items that were not appropriate for adolescent age (e.g., unemployment, subjects sacked from job) were modified to developmentally appropriate items for adolescents. The 12 items used in this study were: serious illness or injury to subject, serious illness or injury to a close relative, death of a parent, death of a close relative, broke off a steady relationship, serious problem with a close friend or relative, subjects suspended or expelled from school, subjects bullied, change of residence, major financial crisis, problem with police and court appearance, and something valuable lost or stolen. Each life event was scored dichotomously (yes=1/no=0). The total score ranges from 0 to 12 .

\section{Children's Depression Inventory}

The Children's Depression Inventory (CDI) is a self-rated, symptom-oriented scale that is suitable for youth aged 7 to 17. There are 27 items quantifying symptoms such as depressed mood, hedonic capacity, vegetative functions, selfevaluation, and interpersonal behaviors. Each CDI item consists of three statements that are rated on a scale that ranges from $0-2$. The total score ranges from 0 to 54 . A score of 25 or greater was regarded as a clinically significant degree of depression. ${ }^{27)}$

\section{The revised Children's Manifest Anxiety Scale}

The revised Children's Manifest Anxiety Scale (RCMAS) is a 37-item, self-report instrument that assesses the severity and nature of anxiety. A response of "Yes" indicates that the item describes the subject's feelings or actions, while a response of "No" indicates that the symptom is not present. The total anxiety score was computed based on 28 items, with a score of 19 or greater suggesting a clinically significant degree of anxiety. ${ }^{28)}$

\section{Statistical analyses}

Cronbach's alphas were calculated to explore the internal consistency of the ETISR-SF. Pearson correlation analysis was used to determine test-retest reliability of the ETISR-SF. Independent t-test was used to examine the group differences in the ETISR-SF. To examine the convergent and divergent validity of the ETISR-SF, Pearson correlations were calculated with several measures, including the CDI, RCMAS, and LTE-Q. SPSS version 21.0 (IBM Corp., Armonk, NY, USA) was used to perform all statistical analyses. A pvalue less than 0.05 was considered significant.

\section{RESULTS}

Table 1 presents the frequency of each traumatic event, item-total correlation, and Cronbach's alphas (minus item). The most common traumatic events to which the subjects were exposed were 'being hit with a thrown object,' 'punched or kicked,' and 'slapped in the face.' The coefficient alpha for the entire scale (0.803) was high.

Table 2 shows the group differences in each domain and total scores of the ETISR-SF. Compared with healthy adolescents, adolescents with depressive disorder scored higher on the ETISR-SF total scale $(\mathrm{t}=2.05, \mathrm{p}=0.044)$ and physical abuse subscale ( $\mathrm{t}=2.03, \mathrm{p}=0.046)$.

Table 3 shows Pearson correlations among the subscales 
of the ETISR-SF. The correlations between the ETISR-SF total and its subscales were moderate to high. The ETISR-SF total and its subscales demonstrated high test-retest reliabilities over a 2-month period. All subscales were temporarily stable.

Table 4 shows Pearson correlations of the ETISR-SF with several measures, including the CDI, RCMAS, and LTE-Q. The ETISR-SF total and its subscale scores were correlated higher with the scores on the LTE-Q than with the scores on the CDI or RCMAS.

\section{DISCUSSION}

Our findings indicate that the Korean version of the ETISR$\mathrm{SF}$ is a reliable and a valid instrument for the assessment of

Table 1. Frequency of endorsement and item-total correlations for the Early Trauma Inventory Self Report-Short Form ( $\mathrm{n}=86$ )

\begin{tabular}{|c|c|c|c|}
\hline Item & Frequency, \% & Item-total correlation & $\alpha$ (minus item) \\
\hline \multicolumn{4}{|l|}{ General trauma } \\
\hline T1. Natural disaster & 4.7 & 0.089 & 0.804 \\
\hline T2. Serious accident & 8.1 & 0.060 & 0.806 \\
\hline T3. Serious personal injury & 14.0 & 0.370 & 0.794 \\
\hline T4. Serious injury/illness of parent & 8.1 & 0.363 & 0.795 \\
\hline T5. Separation of parents & 27.9 & 0.387 & 0.794 \\
\hline T6. Serious illness/injury of sibling & 2.3 & 0.077 & 0.807 \\
\hline T7. Serious injury of friend & 10.5 & 0.277 & 0.799 \\
\hline T8. Witnessing violence & 24.4 & 0.568 & 0.783 \\
\hline T9. Family mental illness & 4.7 & 0.135 & 0.803 \\
\hline T10. Alcoholic parents & 3.5 & 0.284 & 0.799 \\
\hline T11. Seeing someone murdered & 1.2 & 0.140 & 0.803 \\
\hline \multicolumn{4}{|l|}{ Physical abuse } \\
\hline P1. Slapped in the face & 45.3 & 0.531 & 0.784 \\
\hline P2. Burned with cigarette & 30.2 & 0.312 & 0.798 \\
\hline P3. Punched or kicked & 46.4 & 0.445 & 0.790 \\
\hline P4. Hit with thrown object & 50.0 & 0.532 & 0.784 \\
\hline P5. Pushed or shoved & 40.7 & 0.537 & 0.784 \\
\hline \multicolumn{4}{|l|}{ Emotional abuse } \\
\hline El. Often put down or ridiculed & 15.1 & 0.252 & 0.800 \\
\hline E2. Often ignored or made to feel you didn't count & 34.9 & 0.369 & 0.795 \\
\hline E3. Often told you are no good & 17.4 & 0.541 & 0.785 \\
\hline E4. Most of the time treated in cold or uncaring way & 10.5 & 0.377 & 0.794 \\
\hline E5. Parents fail to understand your needs & 17.4 & 0.328 & 0.797 \\
\hline \multicolumn{4}{|l|}{ Sexual abuse } \\
\hline S1. Touched in intimate parts in way that was uncomfortable & 10.5 & 0.478 & 0.790 \\
\hline S2. Someone rubbing genitals against you & 3.5 & 0.213 & 0.801 \\
\hline S3. Forced to touch intimate parts & 1.2 & 0.352 & 0.800 \\
\hline S4. Someone had genital sex against your will & 1.2 & 0.110 & 0.803 \\
\hline S5. Forced to perform oral sex & 0 & 0.000 & 0.804 \\
\hline S6. Forced to kiss someone in sexual way & 1.2 & 0.200 & 0.802 \\
\hline
\end{tabular}

Table 2. Mean differences of Early Trauma Inventory Self Report-Short Form scores between depressive patients and healthy controls

\begin{tabular}{|c|c|c|c|c|}
\hline & Adolescents with depression $(n=40)$ & Healthy controls $(n=46)$ & $t$ & $n$ \\
\hline & Mean (SD) & Mean (SD) & $\dagger$ & $\mathrm{P}$ \\
\hline Total & $5.25(3.57)$ & $3.65(3.65)$ & 2.05 & 0.044 \\
\hline General trauma & $1.25(1.77)$ & $0.96(1.32)$ & 0.88 & 0.381 \\
\hline Physical abuse & $2.60(1.69)$ & $1.79(2.00)$ & 2.03 & 0.046 \\
\hline Emotional abuse & $1.23(1.40)$ & $0.74(1.08)$ & 1.78 & 0.080 \\
\hline Sexual abuse & $0.18(0.47)$ & $0.17(0.57)$ & 0.01 & 0.992 \\
\hline
\end{tabular}

SD: standard deviation 
Table 3. Inter-subscale correlations and test-retest reliability of the Early Trauma Inventory Self Report-Short Form

\begin{tabular}{|c|c|c|c|c|c|}
\hline & General trauma & Physical abuse & Emotional abuse & Sexual abuse & Test-retest reliability \\
\hline Total score & $0.679(<0.001)$ & $0.765(<0.001)$ & $0.709(<0.001)$ & $0.567(<0.001)$ & $0.776(<0.001)$ \\
\hline General trauma & & $0.221(0.041)$ & $0.269(0.012)$ & $0.396(<0.001)$ & $0.836(<0.001)$ \\
\hline Physical abuse & & & $0.372(<0.001)$ & $0.212(0.050)$ & $0.666(<0.001)$ \\
\hline Emotional abuse & & & & $0.446(<0.001)$ & $0.681(<0.001)$ \\
\hline
\end{tabular}

Pearson's R ( $p$ ) was presented

Table 4. Convergent and divergent validity

\begin{tabular}{llll}
\hline \multicolumn{1}{c}{ ETISR-SF } & \multicolumn{1}{c}{ LTE-Q } & CDI & RCMAS \\
\hline Total score & $0.485(<0.001)$ & $0.165(0.138)$ & $0.347(0.004)$ \\
General trauma & $0.368(0.003)$ & $0.160(0.150)$ & $0.349(0.004)$ \\
Physical abuse & $0.351(0.004)$ & $0.065(0.560)$ & $0.168(0.170)$ \\
Emotional abuse & $0.283(0.022)$ & $0.177(0.111)$ & $0.275(0.023)$ \\
Sexual abuse & $0.259(0.037)$ & $0.029(0.795)$ & $0.069(0.575)$
\end{tabular}

Pearson's R (p) was presented. CDI: Children's Depression Inventory, ETISR-SF: Early Trauma Inventory Self Report-Short Form, LTE-Q: List of Threatening Experiences Questionnaire, RCMAS: the revised Children's Manifest Anxiety Scale

childhood trauma among adolescents. The ETISR-SF showed high levels of internal consistency (Cronbach's alphas=0.803), which was comparable to that reported in the previous study in Korean adult sample (Cronbach's alphas=0.806). ${ }^{24)}$ The test-retest reliability (range: 0.666 to 0.836 ) was slightly lower than that reported in the previous study in Korean adult sample of which the test-retest reliability was 0.766 to 0.844 . $^{24)}$ Longer intervals for test-retest reliability in the present study compared to the previous study (i.e., 2 months vs. 3 weeks) may increase the chances that a change in status could occur.

The prevalence of childhood physical abuse $30.2 \%$ to $50.0 \%$ for each item) in this study was higher than that found in studies in Western countries ( $9 \%$ to $45 \%$ for each item) that also used the ETISR-SF. In contrast, the prevalence of childhood sexual abuse ( $0 \%$ to $10.5 \%$ for each item) was much lower than has been reported for Western countries (14\% to $39 \%$ for each item). This relatively low prevalence of sexual abuse is consistent with previous findings in the Korean adult population (1.6\% to $11.5 \%$ for each item). ${ }^{24)}$ The lower percentages of subjects who experienced childhood sexual abuse in this study could be accounted for either by Confucian family values and a collectivist culture that may be protective. ${ }^{29)}$ Other explanation is that Koreans are more likely to conceal embarrassing experiences such as sexual abuse because of the culture of victim blaming in South Korea. ${ }^{30)}$ In contrast, the higher percentages of subjects who experienced childhood physical abuse in this study may reflect the social acceptability of physical punishment in South Korea. In South Korea, Article 915 of the Civil Act 1958 provides adults the "right to take disciplinary action." Laws against violence and abuse are not generally interpreted as prohibiting corporal punishment of children. Other consideration is that about half of our participants are a clinical sample with depression.
In the present study, the depressive group scored significantly higher than control group, particularly in the physical abuse domain. The present findings suggest that increasing social sensitivity towards the detrimental effects of physical abuse that was deemed as a legitimate method of discipline, may decrease adolescent mental health problems related to traumatic experiences.

The ETISR-SF has shown relatively lower correlations with the measurement of depression and anxiety than with the measurement of life-threatening experiences, which suggests that the instrument measures traumatic experiences, rather than assessing the clinical symptoms. However, the correlations of the ETISR-SF total, general trauma, and emotional abuse scores with anxiety were still significant. The observed association between childhood trauma and clinical anxiety symptoms is consistent with previous studies. ${ }^{2,3,-7)}$ Contrary to the well-known association between sexual abuse and the risk of psychiatric problems, ${ }^{4,17)}$ in this study, the relationship of sexual abuse and clinical symptom measures was weaker than that for other types of trauma with clinical symptom measures. This may be due to a very low frequency of reports of sexual abuse in our sample.

The limitation of this study is the relatively small sample size. Moreover, the design of this study was cross-sectional, making identification of a causal relationship between childhood trauma and current clinical symptoms impossible. Lastly, because the subjects had to recall trauma that they had experienced in the past, the respondents' reports of trauma may be prone to inaccuracies, although this is less likely in an adolescent sample than in an adult population. In addition, recall of early trauma might be influenced by affective symptoms as well as memory. 


\section{CONCLUSION}

The results of this study indicate the Korean version of the ETISR-SF appears to be a reliable and valid instrument for the measurement of reported childhood trauma among adolescent. Future prospective studies with a larger sample are needed to measure the accurate traumatic events and to clarify the causal relationships between childhood trauma and clinical symptoms.

\section{Acknowledgments}

This work was supported by the National Research Foundation of Korea (NRF) Grant funded by the Korean Government (MSIP) (NRF2013R1A1A3008158 and NRF- 2014R1A1A3049818).

\section{Conflicts of Interest}

The author has no financial conflicts of interest.

\section{REFERENCES}

1) Bremner JD, Vermetten E, Mazure CM. Development and preliminary psychometric properties of an instrument for the measurement of childhood trauma: the Early Trauma Inventory. Depress Anxiety 2000;12:1-12.

2) Greger HK, Myhre AK, Lydersen S, Jozefiak T. Previous maltreatment and present mental health in a high-risk adolescent population. Child Abuse Negl 2015;45:122-134.

3) McLean CP, Morris SH, Conklin P, Jayawickreme N, Foa EB. Trauma characteristics and posttraumatic stress disorder among adolescent survivors of childhood sexual abuse. J Fam Violence 2014;29: 559-566.

4) Gover AR. Childhood sexual abuse, gender, and depression among incarcerated youth. Int J Offender Ther Comp Criminol 2004;48:683696.

5) Helweg-Larsen K, Frederiksen ML, Larsen HB. Violence, a risk factor for poor mental health in adolescence: a Danish nationally representative youth survey. Scand J Public Health 2011;39:849-856.

6) McLaughlin KA, Greif Green J, Gruber MJ, Sampson NA, Zaslavsky AM, Kessler RC. Childhood adversities and first onset of psychiatric disorders in a national sample of US adolescents. Arch Gen Psychiatry 2012;69:1151-1160.

7) Mills R, Scott J, Alati R, O'Callaghan M, Najman JM, Strathearn L. Child maltreatment and adolescent mental health problems in a large birth cohort. Child Abuse Negl 2013;37:292-302.

8) Kendler KS, Bulik CM, Silberg J, Hettema JM, Myers J, Prescott CA. Childhood sexual abuse and adult psychiatric and substance use disorders in women: an epidemiological and cotwin control analysis. Arch Gen Psychiatry 2000;57:953-959.

9) Molnar BE, Buka SL, Kessler RC. Child sexual abuse and subsequent psychopathology: results from the National Comorbidity Survey. Am J Public Health 2001;91:753-760.

10) Mullen PE, Martin JL, Anderson JC, Romans SE, Herbison GP. Childhood sexual abuse and mental health in adult life. Br J Psychiatry 1993;163:721-732.

11) McLaughlin KA, Green JG, Gruber MJ, Sampson NA, Zaslavsky AM, Kessler RC. Childhood adversities and adult psychiatric disorders in the national comorbidity survey replication II: associations with persistence of DSM-IV disorders. Arch Gen Psychiatry 2010;67: 124-132.

12) Edwards VJ, Holden GW, Felitti VJ, Anda RF. Relationship between multiple forms of childhood maltreatment and adult mental health in community respondents: results from the adverse childhood experiences study. Am J Psychiatry 2003;160:1453-1460.

13) Finkelhor D, Ormrod RK, Turner HA. Polyvictimization and trauma in a national longitudinal cohort. Dev Psychopathol 2007;19:149166.

14) Kessler RC, Davis CG, Kendler KS. Childhood adversity and adult psychiatric disorder in the US National Comorbidity Survey. Psychol Med 1997;27:1101-1119.

15) van der Kolk BA, Fisler RE. Childhood abuse and neglect and loss of self-regulation. Bull Menninger Clin 1994;58:145-168.

16) Afifi TO, MacMillan HL, Boyle M, Taillieu T, Cheung K, Sareen J. Child abuse and mental disorders in Canada. CMAJ 2014;186:E324E332.

17) Chen LP, Murad MH, Paras ML, Colbenson KM, Sattler AL, Goranson EN, et al. Sexual abuse and lifetime diagnosis of psychiatric disorders: systematic review and meta-analysis. Mayo Clin Proc 2010;85:618-629.

18) Zhang ZH, Hao JH, Yang LS, Zhang XJ, Sun YH, Hu CL, et al. [The relationship between emotional, physical abuse and Internet addiction disorder among middle school students]. Zhonghua Liu Xing Bing Xue Za Zhi 2009;30:115-118.

19) Dalbudak E, Evren C, Aldemir S, Evren B. The severity of Internet addiction risk and its relationship with the severity of borderline personality features, childhood traumas, dissociative experiences, depression and anxiety symptoms among Turkish university students. Psychiatry Res 2014;219:577-582.

20) Cater AK, Andershed AK, Andershed H. Youth victimization in Sweden: prevalence, characteristics and relation to mental health and behavioral problems in young adulthood. Child Abuse Negl 2014;38:1290-1302.

21) Park S, Hong JP, Bae JN, Cho SJ, Lee DW, Lee JY, et al. Impact of childhood exposure to psychological trauma on the risk of psychiatric disorders and somatic discomfort: single vs. multiple types of psychological trauma. Psychiatry Res 2014;219:443-449.

22) Denton R, Frogley C, Jackson S, John M, Querstret D. The assessment of developmental trauma in children and adolescents: a systematic review. Clin Child Psychol Psychiatry 2017;22:260-287.

23) Bremner JD, Bolus R, Mayer EA. Psychometric properties of the Early Trauma Inventory-self report. J Nerv Ment Dis 2007;195:211218.

24) Jeon JR, Lee EH, Lee SW, Jeong EG, Kim JH, Lee D, et al. The early trauma inventory self report-short form: psychometric properties of the Korean version. Psychiatry Investig 2012;9:229-235.

25) Kim YS, Cheon KA, Kim BN, Chang SA, Yoo HJ, Kim JW, et al. The reliability and validity of Kiddie-Schedule for Affective Disorders and Schizophrenia-Present and Lifetime version- Korean version (K-SADS-PL-K). Yonsei Med J 2004;45:81-89.

26) Brugha TS, Cragg D. The list of threatening experiences: the reliability and validity of a brief life events questionnaire. Acta Psychiatr Scand 1990;82:77-81.

27) Bang YR, Park JH, Kim SH. Cut-off scores of the Children's Depression Inventory for screening and rating severity in Korean adolescents. Psychiatry Investig 2015;12:23-28.

28) Choi JS, Cho SC. Assessment of anxiety in children: raliability and validity of revised children's manifest anxiety scale. J Korean Neuropsychiatr Assoc 1990;29:691-702.

29) Finkelhor D, Ji K, Mikton C, Dunne M. Explaining lower rates of sexual abuse in China. Child Abuse Negl 2013;37:852-860.

30) Abrams D, Viki GT, Masser B, Bohner G. Perceptions of stranger and acquaintance rape: the role of benevolent and hostile sexism in victim blame and rape proclivity. J Pers Soc Psychol 2003;84:111125. 